\title{
Investigação e Prática em Psicologia Positiva
}

Research and Practice in Positive Psychology Investigación y Práctica en Psicología Positiva

Rita Manuela de Almeida Barros Instituto Piaget - Portugal

José Ignacio Guinaldo Martín Universidade de Aveiro - Portugal

José Fernando Vasconcelos Cabral Pinto Instituto Piaget - Portugal
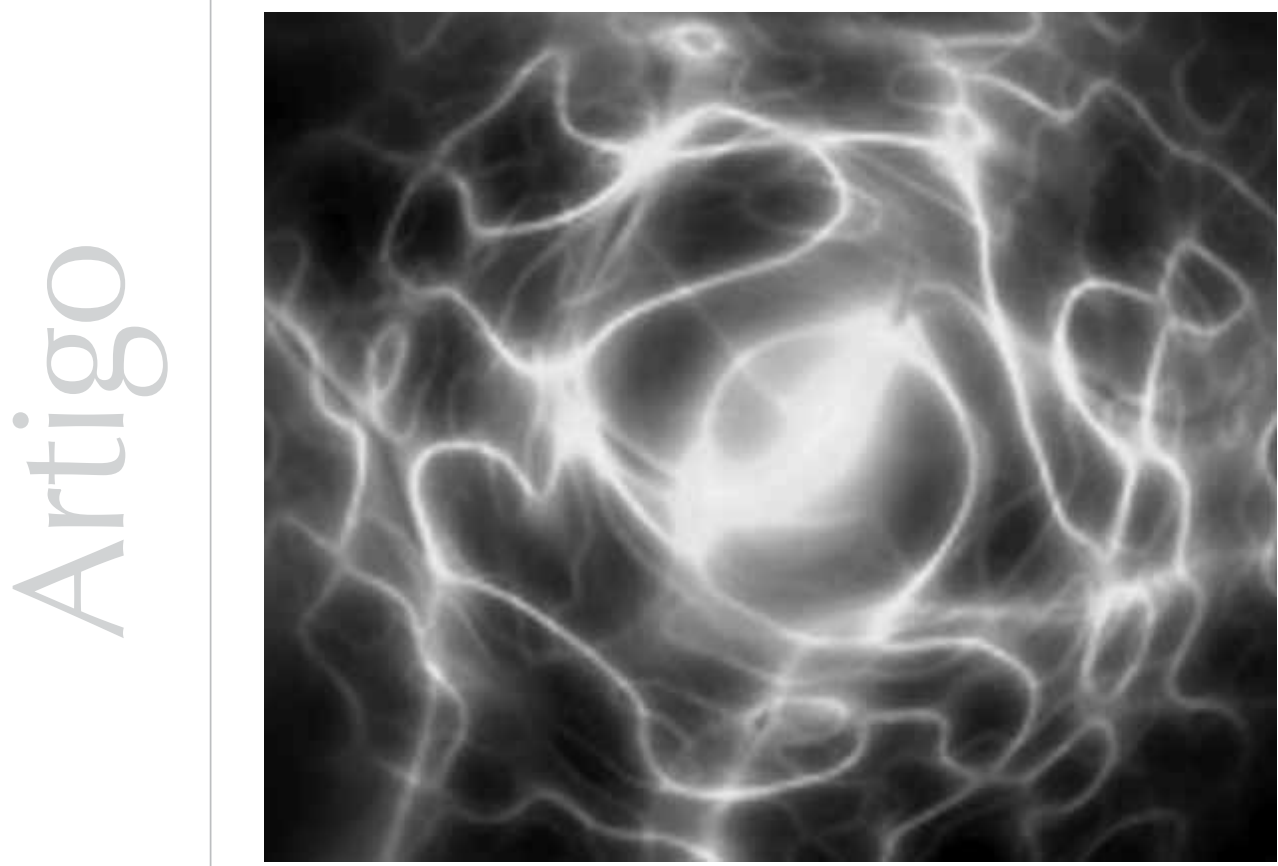
Resumo: Volvida uma década desde a emergência da Psicologia positiva, o presente artigo constitui uma reflexão e síntese desse movimento em expansão. Focámo-nos no seu objecto de estudo e nos aspectos metodológicos que têm caracterizado as investigações nesse domínio, sublinhando tanto as limitações encontradas como as potencialidades e as inovações bem-sucedidas. Procedemos à análise da aplicação prática da Psicologia positiva em múltiplos domínios de intervenção. Situando a Psicologia positiva no cenário científico mundial através da identificação de alguns grupos de trabalho em actividade, reflectimos sobre aspectos que projectam um possível futuro para esse movimento, pautado pela integração de complexidade. Palavras-chave: Psicologia positiva. Investigação. Prática. Grupos de trabalho.

Abstract: After a decade since the emergence of positive psychology, the present article is drawn on a reflection and synthesis of this crescent movement. We have focused on this subject of study and in methodological issues that have characterised the research in this domain, underlying the strengths, weaknesses and well succeeded innovations. We analyze the practical applications of positive psychology in multiple areas of intervention. We placed positive psychology within the world's scientific scene by identifying the currently active working groups and we reflected and forecasted a possible future of this movement, based on integrity and complexity.

Keywords: Positive psychology. Research. Practice. Active working groups.

Resumen: Transcurrida una década desde la emergencia de la Psicología positiva, el presente artículo constituye una reflexión y síntesis de ese movimiento en expansión. Nos enfocamos en su objeto de estudio y en los aspectos metodológicos que han caracterizado las investigaciones en ese dominio, subrayando tanto las limitaciones encontradas como las potencialidades y las innovaciones con final positivo. Procedemos al análisis de la aplicación práctica de la Psicología positiva en múltiples dominios de intervención. Situando la Psicología positiva en el escenario científico mundial a través de la identificación de algunos grupos de trabajo en actividad, reflexionamos sobre aspectos que proyectan un posible futuro para ese movimiento, pautado por la integración de complejidad.

Palabras clave: Psicología positiva. Investigación. Práctica. Grupos de trabajo.

A Psicologia positiva é um campo de análise definido pelo estudo das potencialidades, motivações e realizações humanas (Sheldon \& King, 2001), e analisa as condições que contribuem para a promoção do funcionamento óptimo de pessoas, grupos e instituições (Gable \& Haidt, 2005; Seligman \& Csikszentmihalyi, 2001; Seligman, Steen, Park, \& Peterson, 2005).

De um ponto de vista individual, a Psicologia positiva estuda os processos cognitivos e emocionais subjacentes às experiências subjectivas e aos traços de personalidade dos sujeitos, no sentido de facilitar mudanças comportamentais que se traduzam em estratégias de coping e competências promotoras das potencialidades individuais (Carr, 2007). As experiências subjectivas são analisadas em função do continuum temporal da trajectória de vida dos sujeitos. Alguns estudos exploram as experiências subjectivas passadas, designadamente o bem-estar e a satisfação pessoal (Fujita \&
Diener, 2005), outros analisam o momento presente, centrando-se na felicidade (Diener \& Seligman, 2002) ou nas experiências de fluxo (Csikszentmihalyi, 1990), e outros ainda dedicam-se às projecções dos sujeitos no futuro, dissecando aspectos como a esperança (Abi-Hashem, 2001) ou o optimismo (Peterson, 2000).

A análise individual retrata os traços individuais positivos (Martí, 2006; Seligman et al., 2005), alguns deles relativamente novos em termos de evolução conceptual (Furnham \& Petrides, 2003), de que são exemplo o altruísmo, a espiritualidade, a criatividade ou a coragem (Oliveira, 2004).

De um ponto de vista grupal, a Psicologia positiva estuda as virtudes cívicas e institucionais que promovem nos indivíduos maior adequação em termos de cidadania, responsabilidade social, altruísmo, moderação, tolerância e postura ética (Martí, 2006). 
A Psicologia positiva apresentase com um carácter descritivo, informando os sujeitos nas suas escolhas ao longo da vida, mas nunca definindo trajectórias ideais ou desejáveis (Seligman \& Csikszentmihalyi, 2001).
A Psicologia positiva preenche uma importante lacuna do conhecimento, revelando preocupações na aplicação dos métodos científicos à complexidade do comportamento humano e dotando as investigações de um suporte científico sustentável (Gable \& Haidt, 2005; Sheldon \& King, 2001).

O movimento da Psicologia positiva emergiu como reacção à supremacia da abordagem psicopatológica que dominou a investigação no pós II Guerra Mundial (Seligman \& Csikszentmihalyi, 2001), e os seus actuais contributos mostram que as potencialidades humanas têm um papel extremamente relevante na prevenção da doença, não apenas na dimensão física mas também na psicológica, nomeadamente no que diz respeito aos grandes distúrbios emocionais (Fredrickson, 2001).

Embora a Psicologia positiva enfatize os aspectos positivos do funcionamento da personalidade, as circunstâncias e o momento temporal podem fazer com que os aspectos positivos possam ser nefastos e os aspectos aparentemente negativos sejam funcionais (Diener, Colvin, Pavot, \& Allman, 1991; Held \& Bohart, 2002). Muitos dos aspectos retratados nas investigações, como a tristeza ou a ansiedade (Lourenço, 2000), são apresentados com uma conotação negativa, sem que haja referência às suas funções positivas de adaptação imediata da pessoa enquanto espécie (Fredrickson, 2005).

A Psicologia positiva apresenta-se com um carácter descritivo, informando os sujeitos nas suas escolhas ao longo da vida, mas nunca definindo trajectórias ideais ou desejáveis (Seligman \& Csikszentmihalyi, 2001). Ora, se assim é, mais uma vez terá que se compreender a designação de Psicologia positiva, pois, se a Psicologia pretende ser factual, isto é, direccionada aos fenómenos em si e não ao modo como os fenómenos deveriam ser, então serão igualmente importantes, em termos de pesquisa científica, os fenómenos rotulados de positivos e negativos (Lourenço, 2000).

A Psicologia positiva vai ao encontro da proposta de autorrealização das potencialidades humanas prevista na Psicologia humanista (Bohart \& Greening, 2001; Shapiro, 2001), preconiza a actividade do sujeito orientada para a auto-organização, tal como o construtivismo (Mahoney, 2005), e constructos tradicionalmente abordados na Psicologia da saúde (Pais Ribeiro, 2005), como coping, hardiness ou resiliência, são igualmente alvo de interesse em termos de pesquisa, pelo que a interface entre esses dois domínios tem sido alvo de reflexão (Calvetti, Muller, \& Nunes, 2007).

\section{Investigação em Psicologia Positiva}

Considerando que a Psicologia positiva se traduz em um movimento relativamente recente da comunidade científica, há necessidade de encorajar mais investigações científicas que sustentem modelos teóricos explicativos dos processos e constructos alvo de estudo (Seligman \& Csikszentmihalyi, 2001).

A investigação que tem sido realizada tem-se pautado por alguns aspectos metodológicos que iremos descrever, apontando limitações mas também potencialidades para o futuro, designadamente no que concerne aos instrumentos de avaliação e aos métodos de recolha de dados, aos campos de trabalho e linhas de investigação, aos procedimentos, à amostragem, aos sistemas de controlo de variáveis, à análise de dados e às inovações em termos de tratamento de dados.

$\mathrm{Na}$ literatura, encontram-se muitos estudos destinados à construção de instrumentos de avaliação (e.g., Pavot \& Diener, 1993; Pavot, Diener, \& Suh, 1998), mas a investigação em Psicologia positiva tem-se desenvolvido, 
maioritariamente, a partir de escalas de autorrelato. Considerando a natureza eminentemente subjectiva dos constructos avaliados, encontra-se a prevalência de instrumentos genéricos constituídos por um conjunto relativamente pequeno de itens (escalas multi-item) em relação aos quais os sujeitos se posicionam em uma escala de Likert (Diener \& Oishi, 2005). Menos frequentes são as investigações que se sustentam em escalas com um único item (e.g. Diener \& Diener, 1996; Fujita \& Diener, 2005) ou, pelo contrário, em questionários muito extensos, de que é exemplo o VIA Inventory of Strenghts, com 245 itens (Peterson \& Seligman, 2004). As escalas com um único item mostram-se válidas e adequadas quando se pretende uma medida breve e global (e.g. Davern, Cummings, \& Stokes, 2007), apresentam-se convergentes com outras medidas que avaliam o mesmo constructo e não são muito contaminadas por processos de desejabilidade social (Diener, 1984), embora não seja possível avaliar a sua consistência interna. Essas escalas apresentam, em todo o caso, menor validade e fiabilidade do que as escalas multi-item (Diener et al., 2005).

Embora, face às múltiplas possibilidades que se oferecem à Psicologia positiva, seja necessário desenvolver formas de avaliação que ultrapassem os autorrelatos, como o recurso a outras fontes privilegiadas de informação ou medidas de carácter comportamental, sustentadas nos níveis de saúde ou de produtividade no trabalho (Seligman et al., 2005), parece que o recurso a escalas de autopreenchimento se afigura uma boa opção, dado que essas medidas convergem com outras formas de avaliação, como sejam as entrevistas, o registo de memórias de acontecimentos de vida, os relatos de familiares ou amigos ou ainda as medidas de amostragem experimental (Diener et al., 2005).
Os constructos tradicionalmente pesquisados no domínio da Psicologia positiva (e.g. bem-estar, felicidade ou optimismo) são analisados em diferentes campos de trabalho. Alguns estudos centram-se no impacto de variáveis sociodemográficas, como a idade e o sexo (e.g. Mroczek \& Kolarz, 1998) e as condições socioculturais e relacionais (e.g. Diener \& Oishi, 2005). Outros analisam a influência dos aspectos económico-materiais (e.g. Csikszentmihalyi, 1999) e cognitivomotivacionais (e.g. Lyubomirsky, 2001). Encontram-se também estudos que articulam as dimensões cognitivas e emocionais, como, por exemplo, aqueles que se centram na inteligência emocional (Furnham \& Petrides, 2003), e os que articulam as emoções positivas à organização cognitiva (em termos de atenção, criatividade e flexibilidade de pensamento) e, em consequência, ao aumento de neurotransmissores como a dopamina (Fredrickson, 2001). Os traços de personalidade são também estudados (e.g. DeNeve \& Cooper, 1998), assim como os factores hereditários (e.g. Tellegen et al., 1988).

A pesquisa no domínio da Psicologia positiva tem recorrido a alguns procedimentos inovadores. As investigações sustentadas em registos on-line, nas quais os sujeitos são levados a identificar ou a relatar as emoções vividas no momento presente (Diener, 2000), de que é exemplo o experience sampling method (ESM), permitem avaliar e diferenciar as emoções através de registos on-line realizados em diferentes timings, quer em termos de intensidade como de frequência (Diener \& Oishi, 2005; KimPrieto, Diener, Tamir, Scollon, \& Diener, 2005). O recurso à internet como meio de recolha de dados parece afigurar-se como uma possibilidade tão viável como a recolha de dados tradicional. Encontram-se vários questionários que permitem recolher dados de grandes amostras, ao mesmo tempo que facultam a construção automática de bases de 
dados, com menos dispêndio de tempo para a equipa de investigação e menos sujeitas a erros humanos (Seligman et al., 2005).

Considerando que, salvo algumas excepções (e.g. Diener \& Seligman, 2002; Kim-Prieto et al., 2005), são ainda escassos os estudos que recorrem em simultâneo a várias estratégias e instrumentos de recolha de dados, parece importante que a investigação aposte nesse aspecto. Dessa forma, será possível encontrar eventuais divergências com significado e insights para a compreensão dos fenómenos alvo de estudo da Psicologia positiva (Diener, 2000), de que é exemplo o bem-estar subjectivo (Galinha, 2008).

No domínio da Psicologia positiva, são comuns os estudos com amostras de grande dimensão, muitas vezes representativas de determinadas populações, e estudos transculturais realizados em múltiplos países (e.g. Diener \& Diener, 1996; Diener, 2000). Ainda assim, muitas das amostragens são realizadas por conveniência, pelo que se privilegiam em investigações futuras métodos aleatórios e amostragem (Seligman et al., 2005).

O carácter subjectivo dos processos e variáveis subjacentes à Psicologia positiva requer um sistema de controlo das mesmas. Uma questão que se coloca de imediato diz respeito à desejabilidade social inerente aos dados recolhidos (Prieto-Úrsua, 2006). No entanto, os resultados das investigações encorajam o recurso às escalas de autorrelato, pois os efeitos da desejabilidade social parecem ser irrelevantes (Diener, 1984). Outros autores (e.g. Sink, 2000) mostram-se cépticos em relação às abordagens quantitativas, à redução da experiência fenomenológica a descrições objectivas e à adequação das metodologias correlacionais lineares em fenómenos como a felicidade e o bem-estar. O recurso a abordagens qualitativas e os desenhos de investigação intraindividuais podem ajudar os investigadores a identificar as qualidades que definem a unicidade de cada sujeito (McLafferty \& Kirylo, 2001).

No que diz respeito à análise de dados, Lucas, Diener e Suh (1996), em artigo centrado na validade discriminativa de medidas de bemestar, davam conta da falta de análises com recurso a matrizes multitraço ou multimétodo ou análises factoriais confirmatórias para ilustrar a discriminação entre os componentes afectivos e cognitivos do bem-estar subjectivo. Da mesma forma, estavam ausentes avaliações empíricas que discriminassem o bem-estar subjectivo de outros constructos como a autoestima ou o optimismo, que, embora parecessem constructos diferenciados, poderiam reflectir a mesma disposição de base. Volvida mais de uma década desde a referida publicação, fazem-se sentir as mesmas necessidades, designadamente estudos sustentados em matrizes multitraço e multimétodo e ainda em modelos de regressão para determinados scores (e.g., de bem-estar) a partir de outros constructos (Ryan \& Deci, 2001).

Desenvolvimentos metodológicos mais recentes permitem estabelecer comparações conceptuais, não apenas entre constructos mas também entre modelos teóricos que oferecem diferentes níveis de análise, permitindo colocar novas questões e abrindo portas em termos de investigação futura (e.g. Davern, Cummings, \& Stokes, 2007).

A inclusão de novos métodos estatísticos para analisar a equivalência dos constructos psicológicos em diferentes culturas, assim como a aposta em modelos estruturais, que permitem avaliar em que medida as qualidades psicométricas de um constructo podem ser equacionadas de forma equivalente em diferentes populações, constituem um imperativo para que as conclusões retiradas dos estudos levados a cabo em diferentes culturas possam ser válidas e confiáveis (Ryan \& Deci, 2001). 
O futuro da investigação no domínio da Psicologia positiva deverá apostar em uma perspectiva desenvolvimental (Diener et al., 2005), identificando os padrões de mudança do seu objecto de estudo, como acontece em algumas investigações (e.g. Mroczek \& Kolarz, 1998). Essa perspectiva desenvolvimental não tem sido muito estudada (Seligman et al., 2005), pelo que são desejáveis enquadramentos teóricos que sublinham a dimensão temporal na compreensão de constructos (e.g. Kim-Prieto et al., 2005). A escassez de estudos longitudinais, com excepções relevantes como o estudo de Fujita e Diener (2005), designadamente os de longa duração, é um sintoma relevante dessa necessidade. A diversificação das estratégias metodológicas (por exemplo, suportadas por entrevistas, através das quais se podem conhecer as representações e os significados atribuídos aos constructos em estudo) e o estudo das inter-relações que as variáveis estabelecem entre si em função de aspectos culturais, históricos e sociopolíticos constituem horizontes de pesquisa bastante promissores para os investigadores desse movimento.

\section{Aplicação prática da Psicologia Positiva}

Uma das maiores preocupações dos investigadores nessa área continua a ser a recolha de evidência empírica para uma prática sustentada e para uma classificação consensual das virtudes e forças humanas (Peterson \& Seligman, 2004).

Globalmente, os resultados em termos de intervenção são bastante satisfatórios, e o futuro da prática profissional afigurase promissor (Snyder \& Lopez, 2005). A Psicologia positiva tem sido usada de forma bem-sucedida para promover mudanças comportamentais nos domínios desenvolvimental, psiquiátrico, de saúde em contextos educacionais, vocacionais e comunitários (Carr, 2007; Snyder \& Lopez, 2005), mas torna-se necessária, ainda assim, uma avaliação mais rigorosa, atenta aos efeitos dos projectos de intervenção a longo prazo (Carr, 2007). As intervenções direccionadas à promoção da felicidade mostram que, embora alguns exercícios (expressão da gratidão, identificação de aspectos positivos na vida, narração de histórias relativas a acontecimentos de vida positivos e significativos e definição das virtudes pessoais mais relevantes) sejam eficazes, para o mesmo autor, os resultados das intervenções podem estar comprometidos se estas forem muito circunscritas do ponto de vista temporal, não respeitando os timings necessários ao desenvolvimento e à consolidação de determinadas competências. Seligman et al. (2005) sugerem intervenções com exercícios diversificados, que tenham um impacto mais ou menos imediato e que sejam facilmente integrados em uma rotina diária. Programas isolados não parecem ter efeitos a longo prazo.

O movimento da Psicologia positiva tem um impacto particularmente significativo no domínio comunitário, pela adopção, por parte dos profissionais, de uma postura ecológica dirigida à modificação dos contextos de vida (Carr, 2007). De facto, a intervenção comunitária assenta em referenciais teóricos, tendo o desenvolvimento humano como objecto e como objectivo da intervenção (Menezes, 2007). Em termos de intervenção, a Psicologia positiva destaca-se em situações de ausência de crise, em que a Psicologia tem uma função promotora do desenvolvimento humano. Pensamos ser esse o grande contributo da Psicologia positiva para a prática profissional dos psicólogos que se encontram a intervir em comunidades e instituições comunitárias. A investigação nesse domínio tem sido a mais negligenciada, havendo necessidade de se reverter a situação pelas potencialidades interventivas que se vislumbram (Gable \& Haidt, 2005). 
No domínio da saúde, a Psicologia positiva articula-se com a Psicologia da saúde, não só no coping da doença mas também na prevenção e, especialmente, na promoção da saúde (Calvetti et al., 2007). Veenhoven (2008) sublinha os efeitos da felicidade na saúde física e as consequências dos cuidados preventivos, não só em populações doentes mas também em populações saudáveis. A promoção da saúde pela promoção da felicidade é agora um importante campo de pesquisa. A saúde pública deve assentar em políticas de saúde cujo objectivo seja também o de proporcionar os processos e as condições que conduzam à felicidade. Segundo o autor, a aposta vai para a construção de competências que facilitem a resiliência individual e para as mudanças que ajudem a potencializar os contextos sociais. A educação é também um contexto interventivo privilegiado para a Psicologia positiva. As actuais orientações pedagógicas defendem a personalização da experiência de aprendizagem e a necessidade de construção de significado para a mesma. Os estudos sobre as experiências de fluxo, isto é, as actividades desafiantes que exigem elevadas competências de realização e compromisso, em que a pessoa apresenta total envolvimento e satisfação (Csikszentmihalyi, 1990), são um exemplo paradigmático do contributo das investigações da Psicologia positiva no campo da educação formal e não formal, que também podem ser transpostos para as actividades desportivas e laborais, entre outras. $\mathrm{O}$ mundo global em que vivemos exige mutação sistemática de perfis profissionais, o que faz com que a formação profissional seja também ela mutante e fora do controlo das instituições de formação. A Psicologia positiva pode estar em condições para dar o seu contributo na elaboração de programas de formação, direccionados para a educação geral e para a formação cultural do tipo humanista, dotando-os de estratégias e actividades conducentes à autorrealização dos sujeitos e das comunidades.

Segundo Seligman et al. (2005), as intervenções positivas podem constituir um suplemento às intervenções tradicionais que aliviam o sofrimento humano e poderão traduzir-se no legado prático da Psicologia positiva. Os mesmos autores e Lampropoulos (2001) sugerem que, no futuro, também a psicoterapia incremente as forças humanas. Dessa forma, sublinham-se os contributos da Psicologia positiva para a Psicologia clínica (Calvetti et al., 2007).

\section{A Psicologia positiva no âmbito da Psicologia}

Pode-se afirmar que a Psicologia positiva nasceu nos Estados Unidos da América, dado que é lá que se encontram os seus fundadores, designadamente Seligman e Csikszentmihalyi (2001). No entanto, o interesse por essa área está em franca expansão por todo o mundo. Apresenta-se a Tabela no 1, referente a alguns grupos de trabalho dedicados ao seu estudo e divulgação.

Tabela 1. Grupos de trabalho mais relevantes, dedicados ao estudo e à divulgação da Psicologia

\section{Grupo de trabalho Responsável científico Linhas de trabalho}

Centro de Psicologia Martin Seligman

Positiva da

Universidade da

Pensilvânia (USA)
-Dinamiza intervenções no contexto familiar, escolar, laboral, clínico e comunitário para incrementar o desempenho e o desenvolvimento das potencialidades dos indivíduos e das comunidades.

- Promove a investigação avançada no domínio da Psicologia positiva. 
Continuação tabela 1

\begin{tabular}{|c|c|c|}
\hline Grupo de trabalho & Responsável científico & Linhas de trabalho \\
\hline $\begin{array}{l}\text { Quality of Life } \\
\text { Research Center da } \\
\text { Drucker School of } \\
\text { Management (USA) }\end{array}$ & $\begin{array}{l}\text { Mihaly } \\
\text { Csikszentmihalyi }\end{array}$ & $\begin{array}{l}\text { - Conduz investigações no âmbito da Psico- } \\
\text { logia positiva, designadamente nos domínios } \\
\text { da criatividade e da motivação intrínseca. } \\
\text { - Promove fóruns para os estudantes de todo } \\
\text { o mundo poderem expandir os seus estudos } \\
\text { nesse domínio. }\end{array}$ \\
\hline $\begin{array}{l}\text { Center for Applied } \\
\text { Positive Psychology } \\
\text { (Coventry, England) }\end{array}$ & Alex Linley & $\begin{array}{l}\text { - Promove a investigação avançada no } \\
\text { domínio da Psicologia positiva. } \\
\text { - Cria redes de comunicação nas quais } \\
\text { os investigadores podem partilhar as suas } \\
\text { experiências enquadráveis no âmbito da } \\
\text { Psicologia positiva. } \\
\text { - Apoia o desenvolvimento e a promoção das } \\
\text { suas aplicações. }\end{array}$ \\
\hline $\begin{array}{l}\text { Centre for Confidence } \\
\text { and Well-being } \\
\text { (Glasgow, Scotland) }\end{array}$ & Carol Craig & $\begin{array}{l}\text {-Promove a mudança pessoal, organizacional } \\
\text { e cultural, com base nos resultados das } \\
\text { investigações no âmbito da Psicologia } \\
\text { positiva. } \\
\text { - Organiza conferências, workshops e outros } \\
\text { eventos. }\end{array}$ \\
\hline $\begin{array}{l}\text { European Network for } \\
\text { Positive Psychology } \\
\text { (Europe) - Integra } \\
34 \text { países europeus } \\
\text { que, alternadamente, } \\
\text { coordenam os trabalhos. }\end{array}$ & $\begin{array}{l}\text { Antonella } \\
\text { Delle Fave } \\
\text { (coordenadora } \\
\text { de } 2006 \text { a 2008) }\end{array}$ & $\begin{array}{l}\text { - Promove conferências bianuais, em que são } \\
\text { apresentados os resultados das investigações } \\
\text { de muitos países. } \\
\text { - Reúnem a informação e os dados resultantes } \\
\text { da investigação científica realizada na Europa } \\
\text { no âmbito da Psicologia positiva. }\end{array}$ \\
\hline $\begin{array}{l}\text { International } \\
\text { Positive } \\
\text { Psychology } \\
\text { Association - } \\
\text { Integra cerca } \\
\text { de } 70 \text { países } \\
\text { do mundo que, } \\
\text { alternadamente, } \\
\text { dirigem a } \\
\text { Associação. }\end{array}$ & $\begin{array}{l}\text { Ed Diener } \\
\text { (director até } \\
\text { 2010) }\end{array}$ & $\begin{array}{l}\text { - Promove a ciência e a prática da Psicologia } \\
\text { positiva. } \\
\text { - Pretende constituir um espaço de } \\
\text { comunicação entre os investigadores dessa } \\
\text { área. }\end{array}$ \\
\hline
\end{tabular}

O futuro da Psicologia positiva integra o desafio da complexidade, que não se limita à descrição dos constructos, mas aposta em uma abordagem abrangente das interacções que se estabelecem entre constructos (Gable \& Haidt, 2005). A análise do funcionamento psicológico implica uma perspectiva holística e integradora que permitirá explicar paradoxos como o carácter funcional de emoções e os processos cognitivos negativos em certos sujeitos e em determinados momentos temporais da sua trajectória de vida. Integração e complexidade são os termos-chave para a determinação das relações entre muitos dos aspectos subjacentes ao funcionamento óptimo dos sujeitos, ainda desconhecidos. A construção de modelos empíricos integrativos e abertos tem vindo a ser recentemente sugerida (e.g. Ryan, Huta, \& Deci, 2008). 
Esse movimento foi bem acolhido, talvez pelo momento histórico que se atravessa, devido às necessidades de autorrealização e de construção de um sentido para a vida. O boom de publicações dedicadas a essa temática necessita de um cuidado acrescido quando à sua cientificidade, e cabe aos investigadores que se reveem nesse movimento delimitar aquelas que são pouco sustentadas do ponto de vista empírico e questionáveis do ponto de vista do rigor metodológico e científico.

Por último, deixa-se em aberto uma questão de carácter ético, uma vez que a aposta nos aspectos positivos do funcionamento psicológico e a primazia do bem-estar e da felicidade, enquanto fins últimos da Psicologia positiva, correm o risco de se transformarem em um imperativo e naquilo que alguns autores (e.g. Avía, 2006; Prieto-Úrsua, 2006) designam por "tirania do positivo". Essas e outras questões podem ser inseridas nos currículos de formação académica, possibilitando às novas gerações de psicólogos analisar as limitações e as potencialidades desse movimento (Calvetti et al., 2007).

\section{Conclusão}

Em síntese, podemos concluir que Psicologia positiva apresenta-se como um movimento bastante promissor, na medida em que oferece aos investigadores inúmeros campos de análise, muitos deles ainda por explorar. Como já referimos, a construção de modelos explicativos que permitam estabelecer relações de causalidade entre variáveis e que se afigurem suficientemente abrangentes e integrativos para a compreensão dos complexos fenómenos que são objecto de estudo da Psicologia positiva constitui um desafio para as novas gerações de psicólogos interessados nessas matérias. Será da sua responsabilidade o compromisso de cientificidade e o rigor metodológico que importa imprimir às investigações, no sentido de resguardar a Psicologia positiva e de impedir que a sua designação seja associada a publicações de fraca credibilidade, que em nada dignificam o trabalho sério até agora realizado.

\section{Rita Manuela de Almeida Barros*}

Mestre em Psicologia, Professora adjunta do Instituto Piaget, Canelas - Portugal.

\section{José Ignacio Guinaldo Martín}

Doutorado em Ciências Biomédicas, Professor Auxiliar sem agregação da Universidade de Aveiro - Portugal - Secção

Autónoma de Ciências da Saúde.

E-mail: jmartin@ua.pt

José Fernando Vasconcelos Cabral Pinto

Doutorado em Ciências da Educação, Professor Coordenador da Unidade de Investigação em Educação e Tecnologias

Educativas do Instituto Piaget, Canelas - Portugal.

E-mail: cpinto@gaia.ipiaget.org

* Endereço para envio de correspondência:

Escola Superior de Educação Jean Piaget, Rua António Sérgio, Apartado 551, 4410-269 -

Canelas VNG, Portugal.

E-mail: rbarros@gaia.ipiaget.org

Recebido 29/11/2008, 1a Reformulação, 15/9/2009, Aprovado 30/9/2009.

Abi-Hashem, N. (2001). Rediscovering hope in American psychology. American Psychologist, 56(1), 85-86.

Avía, M. D. (2006). La psicología positiva y la moda de la "soft psychology". Clínica y Salud, 17(3), 239-244.

Bohart, A., \& Greening, T. (2001). Humanistic psychology and positive psychology. American Psychologist, 1(1), 81-82.

Calvetti, P., Muller, M., \& Nunes, M. (2007). Psicologia da saúde e Psicologia positiva: perspectivas e desafios. Psicologia: Ciência e Profissão, 27(4), 706-717.
Carr, E. (2007). The expanding vision of positive behavior support. Journal of Positive Behavior Interventions, 9(1), 3-14.

Csikszentmihalyi, M. (1990). Flow: The psychology of optimal experience. New York: Harper \& Row.

Csikszentmihalyi, M. (1999). If we are so rich, why aren't we happy? American Psychologist, 54(10), 821-827.

Davern, M., Cummings, R., \& Stokes, M. (2007). Subjective wellbeing as an affective-cognitive construct. Journal of Happiness Studies, 8, 429-449. 
DeNeve, K., \& Cooper, H. (1998). The happy personality: A meta-analysis of 137 personality traits and subjective wellbeing. Psychological Bulletin, 124(2), 197-229.

Diener, E. (1984). Subjective well-being. Psychological Bulletin, 95(3), 542-575.

Diener, E., Colvin, C., Pavot, W., \& Allman, A. (1991). The psychic costs of intense positive affect. Journal of Personality and Social Psychology, 61(3), 492-503.

Diener, E., \& Diener, C. (1996). Most people are happy. Psychological Science, 7(3), 181-185.

Diener, E. (2000). Subjective well-being: The science of happiness and a proposal for a national index. American Psychologist, 55(1), 34-43.

Diener, E., \& Seligman, M. (2002). Very happy people. Psychological Science, 13(1), 81-84.

Diener, E., \& Oishi, S. (2005). The nonobvious social psychology of happiness. Psychological Inquiry, 16(4), 162-167.

Fredrickson, B. (2001). The role of positive emotions in positive psychology. American Psychologist, 56(3), 218-226.

Fredrickson, B. (2005). Positive emotions. In C. Snyder \& S. Lopez (Eds.), Handbook of Positive Psychology (pp. 120-134). Oxford: Oxford University Press.

Fujita, F., \& Diener, E. (2005). Life satisfaction set point: Stability and change. Journal of Personality and Social Psychology, 88(1), 158-164

Furnham, A., \& Petrides, K. (2003). Trait emotional intelligence and happiness. Social Behavior and Personality, 31(8), 815-824.

Gable, S., \& Haidt, J. (2005). What (and why) is positive psychology? Review of General Psychology, 9(2), 103-110.

Galinha, I. C. (2008). Bem-estar subjectivo: Factores cognitivos, afectivos e contextuais. Coimbra: Quarteto.

Held, B., \& Bohart, A. (2002). Introduction: The (overlooked) virtues of "unvirtuos" attitudes and behavior - Reconsidering negativity, complaining, pessimism and "false" hope. Journal of Clinical Psychology, 58, 961-964.

Kim-Prieto, C., Diener, E., Tamir, M., Scollon, C., \& Diener, M. (2005). Integrating the diverse definitions of happiness: A time-sequential framework of subjective well-being. Journal of Happiness Studies, 6, 261-300.

Lampropoulos, G. (2001). Integrating psychopathology, positive psychology, and psychotherapy. American Psychologist, 56(1), 87-88.

Lourenço, O. (2000). Orientação para o positivo e para o negativo: um caso especial de assimetria no comportamento humano. Psicologia, Educação e Cultura, 2, 227- 241.

Lucas, R., Diener, E., \& Suh, E. (1996). Discriminant validity of well-being measures. Journal of Personality and Social Psychology, 71(3), 616-628.

Lyubomirsky, S. (2001). Why are some people happier than others? The role of cognitive and motivational processes in well-being. American Psychologist, 56(3), 239-249.

Mahoney, M. (2005). Construtivism and positive psychology. In C. Snyder \& S. Lopez (Eds.), Handbook of positive psychology (pp. 745-750). Oxford: Oxford University Press.

Martí, M. (2006). El estudio científico de las fortalezas transcendentales desde la psicología positiva. Clínica y Salud, $7(3), 245-258$.

McLafferty, C., \& Kirylo, J. (2001). Prior positive psychologists proposed personality and spiritual growth. American Psychologist, 56(1), 84-85.
Menezes, I. (2007). Intervenção comunitária: uma perspectiva psicológica. Porto: Legis.

Mroczek, D., \& Kolarz, C. (1998). The effect of age on positive and negative affect: A developmental perspective on happiness. American Psychologist, 75(5), 1333-1349.

Oliveira, J. (2004). Psicologia positiva. Porto: Edições ASA.

Pais Ribeiro, J. L. (2005). Introdução à psicologia da saúde. Coimbra: Quarteto.

Pavot, W., \& Diener, E. (1993). Review of the satisfaction with life scale. Psychological Assessment, 5(2), 164-172.

Pavot, W., Diener, E., \& Suh, E. (1998). The temporal satisfaction with life scale. Journal of Personality Assessment, 70(2), 340354.

Peterson, C. (2000). The future of optimism. American Psychologist, 55(1) 44-55.

Peterson, C., \& Seligman, M. (2004). Character strengths and virtues: A handbook and classification. Washington, DC: American Psychological Association.

Prieto-Úrsua, M. (2006). Psicología positiva: una moda polémica. Clínica y Salud, 17(3), 319-338.

Ryan, R., \& Deci, E. (2001). On happiness and human potentials: A review of research on hedonic an eudaimonic well-being. Annual Review of Psychology, 52, 141-166.

Ryan, R., Huta, V., \& Deci, E. (2008). Living well: A selfdetermination theory perspective on eudaimonia. Journal of Happiness Studies, 9, 139-170.

Seligman, M., \& Csikszentmihalyi, M. (2000). Positive psychology: An introduction. American Psychologist, 55(1), 5-14.

Seligman, M., \& Csikszentmihalyi, M. (2001). Reply to comments. American Psychologist, 56(1), 89-90.

Seligman, M., Steen, T., Park, N., \& Peterson, C. (2005). Positive psychology: Empirical validation of interventions. American Psychologist, 60(5), 410-421.

Shapiro, S. (2001). Illogical positivism. American Psychologist, 56(1), 82.

Sheldon, K., \& King, L. (2001). Why positive psychology is necessary. American Psychologist, 56(3), 216-217.

Sink, J. (2000). Why can't we measure happiness? American Psychologist, 55(10), 1162-1163.

Snyder, C., \& Lopez, S. (2005). Positive psychology: A declaration of independence. In C. Snyder \& S. Lopez (Eds.), Handbook of positive psychology (pp. 751-767). Oxford: Oxford University Press.

Tellegen, A., Lykken, D., Bouchard, T., Wilcox, K., Segal, N., \& Rich, S. (1988). Personality similarity in twins reared apart and together. Journal of Personality and Social Psychology, 54, 1031-1039.

Veenhoven, R. (2008). Healthy happiness: Effects of happiness on physical health and the consequences for preventive health care. Journal of Happiness Studies, 9, 449- 469. 